\title{
Intrascleral with Intracorneal Cysticercosis: An Unusual Presentation
}

\author{
Patyal S, Uday Y, Kakkar S and Sharma VK*
}

Army Hospital Research and Referral, Delhi, India

*Corresponding author: Vijay K Sharma, Assistant Professor, Ophthalmology Army Hospital Research and Referral, Delhi Cantt, India, Tel: 091-8826956710; E-mail:vijayshrma007@gmail.com

Citation: Patyal S, Uday Y, Kakkar S, Sharma VK (2017) Intrascleral with Intracorneal Cysticercosis: An Unusual Presentation. J Eye Cataract Surg Vol. 3 No.3: 31.

Received date: June 21, 2017; Accepted date: July 17, 2017; Published date: July 20, 2017

Copyright: (C2017 Patyal S, et al. This is an open-access article distributed under the terms of the Creative Commons Attribution License, which permits unrestricted use, distribution, and reproduction in any medium, provided the original author and source are credited.

\section{Abstract}

15 years old male reported with diminution of vision in the left eye of three months duration. He had a cystic lesion in the inferonasal area of the cornea straddling the limbus, partially on the cornea and partially on the sclera. Surgical excision of the lesion revealed it to be a continuous intrascleral and intracorneal cyst. Histopathology confirmed it to be a calcified cysticercosis.

Keywords: Intrascleral; Intracorneal cysticercosis; Swelling

\section{Introduction}

Cysticercosis is the commonest parasitic infection in the world responsible for the highest cause of acquired epilepsy in the world with an estimated prevalence of 50 million infected people. 1 Certain areas in the world especially areas of poverty and poor hygiene, are endemic to the infection including Asia, Central and South America and Africa [1,2]. Ocular cysticercosis is the development of cysticercus in ocular tissues and occurs in $13-46 \%$ of infected patients. The first case of ocular cysticercosis was reported by Sommerring in 1830 and the larva was demonstrated and extracted by Schott in 1836. Cysticercus may be found in various ocular tissues (13-46\%) with highest frequency in the subretinal tissues (35\%) followed by vitreous $(22 \%)$, conjunctiva (22\%), anterior segment $(5 \%)$, and orbit (1\%) $[3,4]$. In a recent case report, ocular cysticercosis presented as neovascular glaucoma [5].

We present the first case report of ocular cysticercosis with simultaneous intrascleral and intracorneal involvement.

\section{Case Report}

A 15-years old male reported with a swelling in the left eye of eleven years duration. The swelling had remained unchanged for the past 11 years and had started increasing in size and colour only in the preceding three months. It was associated with diminution of vision in the left eye since last three months. There was no associated pain, redness or discharge. There was no history of itching, worms in stool, cough, headache or convulsions. He gave no history of prior ocular or systemic treatment. There was no history of nausea, vomiting, headache, lethargy, confusion, weakness, raised lesions on skin and seizures. Detailed systemic evaluation did not reveal any abnormality. Ocular examination revealed uncorrected visual acuity of 20/20 in right eye and 20/60 in left eye improving to $20 / 20$ with $-3.50 D C$ at $180^{\circ}$ degrees. Right eye examination was essentially normal. Left eye examination revealed a cystic swelling approximately $10 \times 10 \mathrm{~mm}$ in the inferonasal area partially in the cornea partially subconjunctival, extending from 5 O'clock to 8 O'clock $h$ straddling the limbus (Figure 1A and 1B).

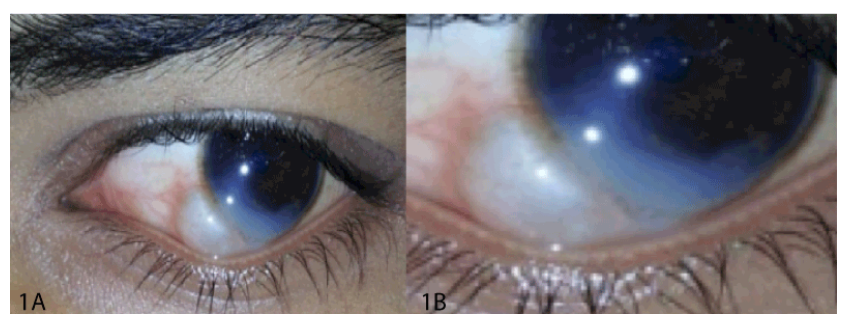

Figure 1: A cystic swelling approximately $10 \times 10 \mathrm{~mm}$ in the inferonasal area partially in the cornea partially subconjunctival, extending from 5 o'clock to 8 o'clock $h$ straddling the limbus.

The swelling bulged more on the conjunctival side than on the corneal and was accompanied by conjunctival congestion. It indented the lower lid resulting in its outward protusion. The colour of both conjunctival and corneal lesion was yellowish white. No particulate matter was seen nor was any movement visible within it on slit lamp examination. Barring the presence of the swelling in the inferonasal area of the cornea, the rest of the cornea was clear. The anterior chamber was of normal depth except inferonasally where the depth and contents could not be determined. The rest of the anterior chamber had no cells, flare or keratic precipitates on the endothelium. Iris was normal in texture and colour, with no evidence of prior uveitis. Fundus did not reveal any abnormality. Intraocular pressure in right eye was 12 and left eye $14 \mathrm{mmHg}$ by applanation tonometry. Ultrasonic biomicroscopy of the left eye revealed small opaque 
subconjunctival shadow in the inferonasal area in the left eye. No cystic lesion was seen Figure 2.

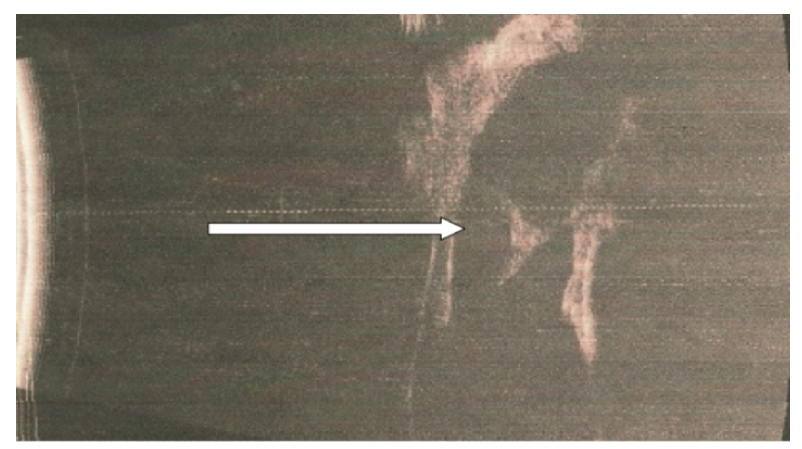

Figure 2: Ultrasonic biomicroscopy of the left eye revealed small opaque subconjunctival shadow in the inferonasal area in the left eye.

All other investigations including haemoglobin, total leucocyte and differential leucocyte counts and absolute eosinophil counts were normal. IgG Ab for cysticercus was: $0.23 \mu / \mathrm{ml}$ (negative for cysticercus). Routine and microscopic examination of stool (repeated thrice) was normal. X-Rays of chest and both thighs were normal. MRI brain and orbits showed no lesion in the brain but revealed a thickening present in the conjunctivo-corneal junction in the inferomedial quadrant of the left eye corresponding to the lesion seen clinically.

Excisional biopsy was performed on under local anaesthesia. An incision was made on the inferior conjunctival border of the cyst and separated from the underlying tissues, but the margins of the cyst could not be delineated. On dissection of the conjunctiva from the swelling, it was found to be intrascleral Figure 3.

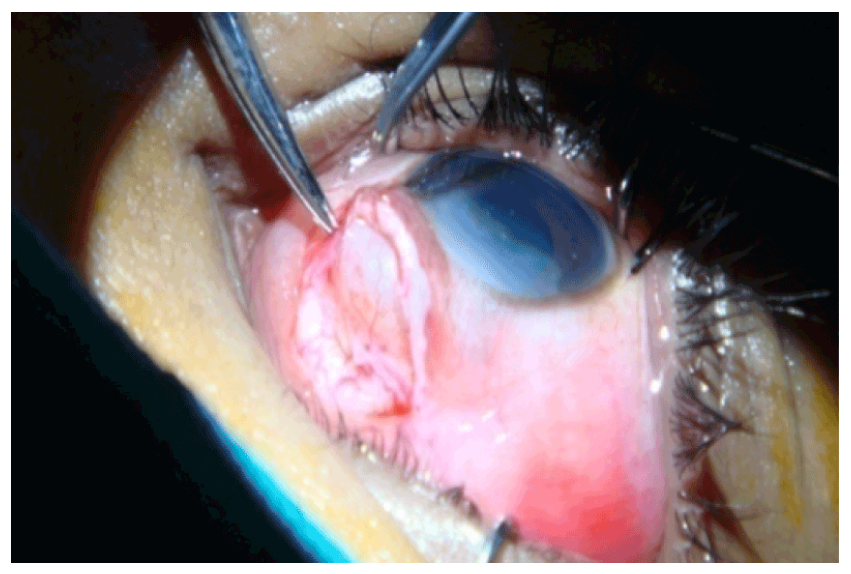

Figure 3: Intrascleral part of the cyst.

A small puncture was made through the inferior end of the swelling and mild pressure was applied to it. Only milky fluid emanated from the swelling which could not be collected. A partial thickness scleral flap was made, with the incision parallel to the first incision. The scleral flap was then excised from the limbus Figure 4.

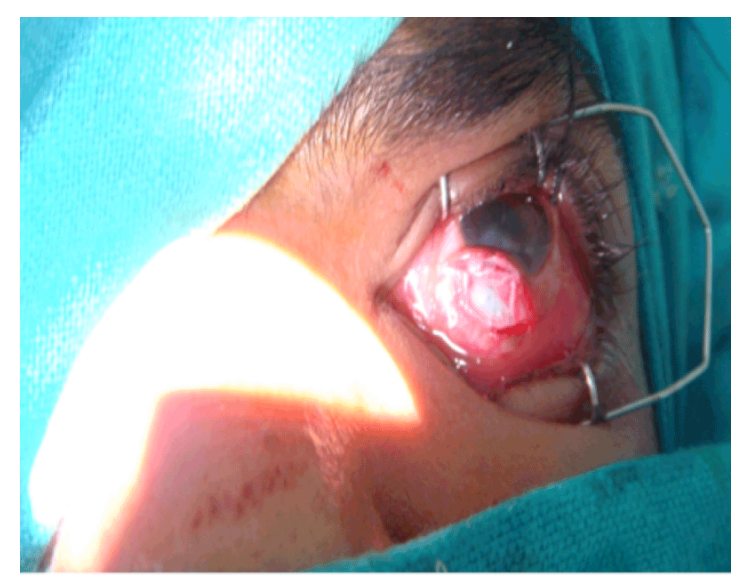

Figure 4: Excision of the anterior flap of the cystic scleral wall.

A small whitish particulate matter was seen adhering to the inner wall of the anterior scleral flap and was sent for histopathology and the report confirmed it to be ocular cysticercosis. Figure $\mathbf{5}$.

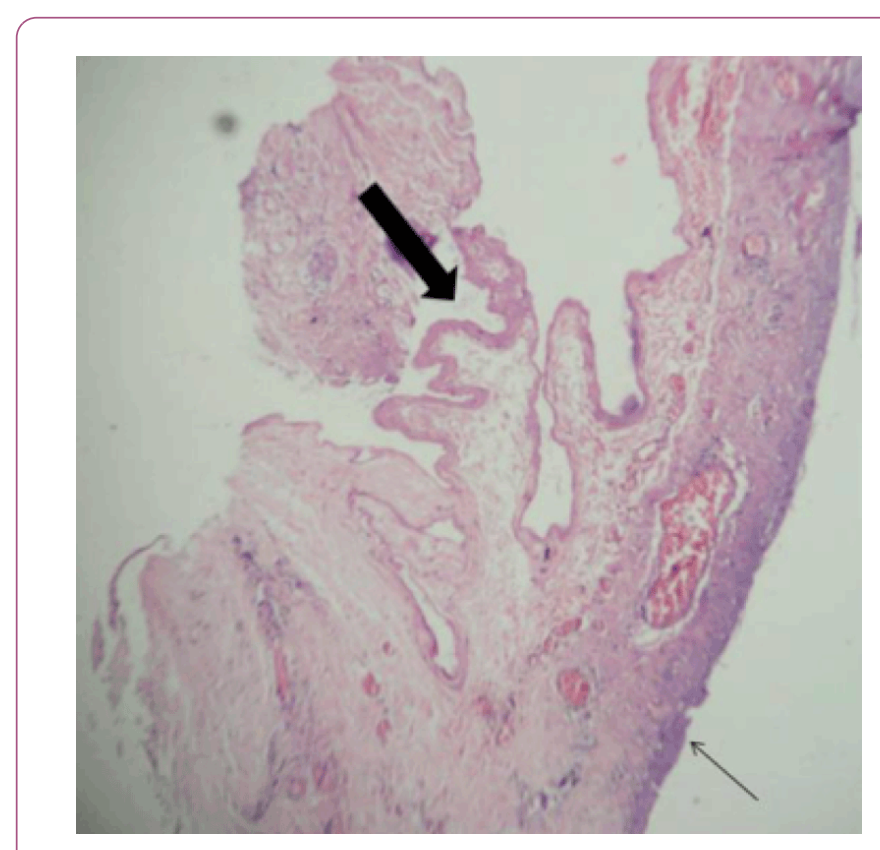

Figure 5: Haematoxylin and Eosin stained section (40x) of part of conjunctival cyst showing part of the thinned out lining of stratified squamous epithelium (thin arrow) and a part of the mummified remnants of the wavy cuticle of the cysticercus (thick arrow).

Residual sclera bed was firm and no underlying bulge was seen. Milky fluid emanated on mild pressure on the corneal side of the swelling which was connected to the scleral half. All residual fluid was evacuated from the corneal side. The residual sclera bed, corneal pocket and the conjunctival sac were washed thoroughly with Ringer Lactate solution. The conjunctival wound was sutured with $8 / 0$ Vicryl sutures.

Postoperatively, the patient was treated with antibiotic steroid combination eye drops, tablet Albendazole 15 
$\mathrm{mg} / \mathrm{kg} /$ per day with tablet Prednisolone $1 \mathrm{mg} / \mathrm{kg}$ body weight for 6 weeks to obviate effects of any cysticercus in any part of the body though all investigations indicated a solitary involvement. The immediate postoperative conjunctival congestion subsided with steroid antibiotic drops. No uveal reaction was seen post operatively Figure 6.

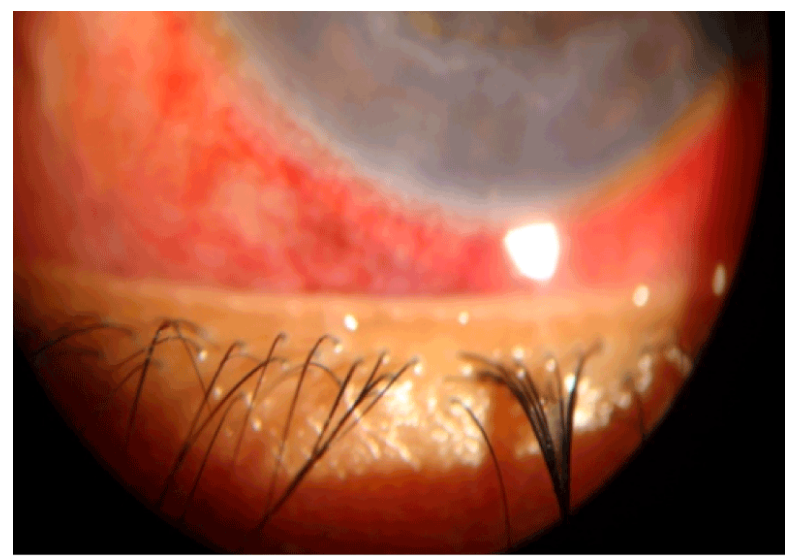

Figure 6: Postoperative photograph taken $72 \mathrm{~h}$ after excision of the cyst.

A Macular grade scar with irregular corneal outline remained on the inferomedial aspect of the cornea. There was no recurrence of the swelling in the follow up period of two years.

\section{Discussion}

Our patient had an unusual presentation of intrascleral with intracorneal cyst which to the best of our knowledge, has not been reported in literature. The case seen by us presented at 15 years of age though his mother gave a history of presence of this swelling in the eye for 11 years. In our patient, the lesion being intrascleral and intracorneal, presented with diminution of vision, a symptom commonly found in patients who have intraocular cysts $[3,6]$. This was possibly due to its intracorneal nature which caused astigmatism and a refractive error as vision improved with pinhole. In our patient, a symptomless swelling was present in the left eye for eleven years other than a cosmetic defect, suggestive of a viable cyst because of which the patient did not seek any treatment. The cyst may then have undergone degeneration resulting in an inflammation and increase in its size in three months. The inflammation spread into the corneal tissue causing the corneal layers to separate resulting in a refractive error and therefore causing diminution of vision.

All investigations in the patient were within normal limits including total eosinophil count and IgG. No other cystic lesion was seen elsewhere in the body including the muscles and brain as evidenced by X- Rays of thigh muscles and MRI of brain, which is one of the most accurate techniques for assessing the degree of infection especially neurocysticercosis [6]. MRI revealed a thickening of tissue on the inferomedial aspect of the anterior surface of left globe with no intracranial lesions.
Seronegativity in this case is possible as it was a single lesion of cysticercosis [1], and a single parasite may not elicit a strong antibody response. The ultrasound biomicroscopy showed a sonolucent area in the inferonasal quadrant of the eye. The reason why a cystic mass was not visible by ultrasonography was determined during surgical excision where a dead cyst was found with surrounding inflammation.

Clinically since the lesion was subconjunctival in location, a conjunctival incision was made inferiorly and on dissecting the conjunctiva off the swelling, it was found to be intrascleral. Therefore, a small puncture wound was made so as to allow evacuation of the contents. Milky fluid emanated from the swelling, showing that degeneration had occurred in the cyst which had invoked an inflammatory reaction with resultant increase in its size in three months.

The calcified remains of the cysticercus which were found on the inner aspect of the superior sclera flap, were proved histopathologically to be cysticercus which can be taken as the sine qua non for clinching the diagnosis. No sex predilection has been found in any study. Younger individuals are more commonly affected- an Indian study by Malik et al. found $68 \%$ of their patients between 10-30 years [7], and Reddy et al. have reported $90 \%$ of their patients to be below 15 years of age [8].

Geographical differences also occur in the sites of localization of cysticercosis. Western literature has reported a greater involvement of the posterior segment with cysticercosis while Indian literature has reported higher numbers in the ocular adnexa [3]. A distinct change in the presentation of ocular cysticercus has been noted in a study from South India, where presentations of subconjunctival lesions have decreased significantly whereas intraocular lesions have shown significant increase in number [3]. One of the reasons for this change is possibly improved medical treatment for lesions in the anterior segment or orbit and adnexa [3]. It has been postulated that the cysts are brought via the posterior ciliary arteries to the subretinal space usually in the region of the posterior pole [9].

Ocular cysticercosis may present in a myriad ways: as a painless enlarging mass in the eyelid, hyperemic epibulbar mass subconjunctivally, cysts in the anterior chamber attached to the iris or the anterior lens capsule or as cysts in the vitreous or subretinal space [9].

Clinical manifestations of ocular cysticercosis depend upon location inside the eye, size, host's immune status and inflammatory reactions [3]. It is believed that degenerating cysts rapidly increase in size due to osmosis, cause compression of surrounding tissues and release antigens into surrounding tissues resulting in an inflammatory response. Viable cysts may not cause any inflammatory response [3].

Treatment of ocular cysticercosis depends upon the site of involvement. It is recommended that surgery should be performed in intraocular cases but cysticercosis is best treated with medical therapy when found deep in the orbit [9]. Preoperative drug therapy is not advocated to prevent death of the parasite which may induce severe inflammatory reaction [10], though in this case degenerative changes had already taken place as found per operatively. 
A large number of studies in the world have described subconjunctival cysticercus which is one of the commonest locations of the parasite in the eye. But intrascleral cysticercus connected to the corneal layers has not been found despite literature search using Yahoo, Google and Pubmed and standard textbooks. This case, to the best of our knowledge, is the first, where an intrascleral cyst was found in continuation with a cyst found between the layers of the cornea.

\section{Conflict of Interest}

Nil.

\section{References}

1. Héctor H García, Armando E Gonzalez, Carlton A W Evans, Robert H Gilman (2003) for the Cysticercosis Working Group in Peru. The Lancet 362: 547-556.

2. Kraft $R$ (2007) Cysticercus: An emerging parasitic disease American Family Physician 76: 92-96.
3. Madigubba S, Vishvanath K, Reddy GBKG, Vemuganti GK (2007) Changing trends in ocular cysticercosis over two decades: An analysis of 118 surgically excised cysts. Indian J Microbiology 25: 214-219.

4. Kruger-Leite, Jalkh AE, Quiroz H, Schepens CL (1985) Intraocular cysticercosis. Am j Ophthalmol 99: 252-257.

5. Ratra D, Phogat C, Singh M, Choudhari NS (2010) Intravitreal cysticercosis presenting as neovascular glaucoma. IJO 58: 70-73.

6. Raval V, Khetan V (2012) Spectral domain optical coherence tomography Features of Subretinal Cysticercus Cyst. J Ophthalmic and Vision Research 7: 347-349.

7. Malik SR, Gupta AK, Choudhary S (1968) Ocular Cysticercosis. Am J Ophthalmol 66: 1168-1171.

8. Reddy PS, Satyendran OM (1964) Ocular cysticercosis. Am J Ophthalmol 57: 664.

9. Kaliaperuma S, Rao VA, Parija SC (2005) Cysticercosis of the eye in South India- A case series. Indian J Microbiology 23: 227-230.

10. Dinesh Patel, Hemkala Trivedi, Sujit Murade, Sujata Tank (2011) Ocular cysticercosis: A review. Bombay Hospital J 53: 420-425. 